\title{
In Vivo Measurement of Longitudinal Chromatic Aberration in Patients Implanted With Trifocal Diffractive Intraocular Lenses
}

\author{
Maria Vinas, PhD; Ana Gonzalez-Ramos, MSc; Carlos Dorronsoro, PhD; Vyas Akondi, PhD; \\ Nuria Garzon, PhD; Francisco Poyales, MD; Susana Marcos, PhD
}

\begin{abstract}
PURPOSE: To measure the longitudinal chromatic aberration (LCA) by both psychophysical methods and in vivo double-pass retinal imaging in patients bilaterally implanted with trifocal diffractive intraocular lenses (IOLs).
\end{abstract}

METHODS: Measurements were performed with a polychromatic adaptive optics system provided with a supercontinuum laser, a Hartmann-Shack wavefront sensor, a deformable mirror, a motorized Badal system, a pupil monitoring system, a double-pass retinal imaging channel, and a psychophysical channel with monochromatically illuminated stimuli. Ten patients (20 eyes) bilaterally implanted with hydrophilic trifocal diffractive IOLs (FineVision POD F; PhysIOL, Liege, Belgium) participated in the study. Measurements were performed in both eyes at three different viewing distances $(0.00$, +1.75 , and +3.50 diopters [D]). Subjective best focus of monochromatic stimuli at five wavelengths (480 to $700 \mathrm{~nm}$ ) was obtained using the Badal system. Best focused images of through-focus double-pass image series were obtained at three wavelengths (480 to 700 $\mathrm{nm}$ ). LCA was computed from chromatic difference of focus curves (objective and subjective) as the difference between 480 and $700 \mathrm{~nm}$ at near, intermediate, and far.

RESULTS: The average subjective LCA was $0.82 \pm$ $0.05 \mathrm{D}$ for far, $0.27 \pm 0.15 \mathrm{D}$ for intermediate, and $0.15 \pm 0.15 \mathrm{D}$ for near. The average objective LCA was $0.72 \pm 0.10 \mathrm{D}$ for far, $0.19 \pm 0.15 \mathrm{D}$ for intermediate, and $0.07 \pm 0.17 \mathrm{D}$ for near.

CONCLUSIONS: Objective LCA was lower than subjective LCA, which was in agreement with previous studies on patients with phakic and monofocal IOLs. In vivo measurements of LCA enable understanding of the relative contribution of refractive and diffractive LCA and will eventually optimize IOL designs to improve polychromatic image quality.

[J Refract Surg. 201X;X(X):XX-XX.]

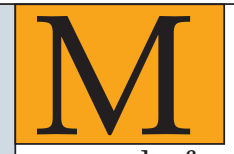

ultifocal corrections are increasingly used solutions for presbyopia, working by the principle of simultaneous vision (ie, projecting simultaneously focused and defocused images on the retina). These corrections, in the form of intraocular lenses (IOLs) or contact lenses, provide multifocality at the expense of reducing optical quality at all distances. Current multifocal IOLs in the market work on diffractive or refractive principles, including refractive bifocal concentric and angular, diffractive bifocal and trifocal, extended depth of focus, or hybrid designs. ${ }^{1}$

On-bench evaluations of diffractive bifocal and trifocal multifocal IOLs ${ }^{2,3}$ are generally performed by measuring throughfocus image quality and modulation transfer function ${ }^{4,5}$ from the light distribution and efficiency of the different foci. ${ }^{6}$ Most on-bench studies are performed in monochromatic light. ${ }^{2,3,5-7}$ On the other hand, visual performance in patients implanted with multifocal IOLs is generally assessed in terms of defocus curves, where visual acuity is measured through-focus, generally using black targets on white backgrounds. HartmannShack or laser ray tracing aberrometry, excellent tools to assess optical quality with monofocal lenses, or smooth refractive multifocal designs, are generally not well suited to measure segmented ${ }^{8}$ or diffractive IOLs, ${ }^{9}$ and only work in monochromatic light. However, polychromatic image quality is of interest because the real world is polychromatic.

From the Instituto de Óptica, Consejo Superior de Investigaciones Científicas, Madrid, Spain (MV, AG-R, CD, VA, SM); and IOA Madrid Innova Ocular, Madrid, Spain (NG, FP).

Submitted: April 10, 2017; Accepted: July 28, 2017

Supported by European Research Council ERC-2011-AdG-294099; Spanish Government FIS2014; collaborative agreement with PhysIOL (Liege, Belgium).

The authors have no financial or proprietary interest in the materials presented herein

The authors thank Laura Barrios from the Scientific Calculus Center of the Spanish National Research Council for help with the statistical analysis within the study.

Correspondence: Maria Vinas, PhD, Instituto de Óptica, Consejo Superior de Investigaciones Científicas, Serrano 121, 28006 Madrid, Spain. E-mail: maria. vinas@io.cfmac.csic.es

doi:10.3928/1081597X-20170814-01 
The replacement of the crystalline lens by an IOL modifies the chromatic dispersion properties of the eye because these are affected by the refractive index wavelength dependency of the IOL material. Therefore, the optical performance of the pseudophakic eye in polychromatic light will be determined by both the IOL material $^{10-12}$ and the IOL design, which is especially relevant in diffractive designs. ${ }^{13}$ The impact of the chromatic aberrations in the pseudophakic eye has been acknowledged $^{14-16}$ and there are few studies reporting in vivo longitudinal chromatic aberration (LCA) of pseudophakic eyes implanted with monofocal IOLs. ${ }^{10,17-19}$ In a previous study from our group, we showed in vivo LCA in patients bilaterally implanted with monofocal aspheric hydrophobic and hydrophilic IOLs, using psychophysical and wavefront-sensing methods in a wide spectral range ( 480 to $700 \mathrm{~nm}$ ). The LCA (400 to $700 \mathrm{~nm}$ ) was 1.37 $\pm 0.08 \mathrm{D}$ (hydrophobic) and $1.21 \pm 0.08 \mathrm{D}$ (hydrophilic) from psychophysical measurements and $0.88 \pm 0.07$ and $0.73 \pm 0.09 \mathrm{D}$ from wavefront-sensing measurements, respectively, confirming the dependence of LCA on the IOL material. ${ }^{20}$

The impact of the chromatic aberrations in the pseudophakic eye has generated interest in correcting the LCA of the eye, and there are even proposals for IOL (diffractive) designs aiming at correcting the ocular LCA. ${ }^{11,21}$ Ravikumar et al. ${ }^{16}$ showed computationally that achromatization by a diffractive IOL provided significant improvement in polychromatic retinal image quality. Diffractive multifocal IOLs in particular have interesting chromatic properties ${ }^{12,22}$ because they are subject to both refractive chromatic aberration and wavelength-dependent diffractive effects. In a recent publication, Millán et al. ${ }^{23}$ evaluated, theoretically and on-bench, the LCA and through-focus energy efficiency of bifocal IOLs of two different designs and materials. They found that the refractive base power of bifocal IOLs increases the positive LCA of prior ocular media for far vision and the achromatizing effect of diffractive bifocal IOLs may compensate, in part, for the natural eye's LCA for near vision.

Trifocal diffractive IOLs were recently developed, aiming at generating an intermediate focal point in the IOL optic to improve optical quality at that distance while maintaining good far and near vision. Some studies have evaluated vision with bilateral implantation of the FineVision POD F IOL (PhysIOL, Liege, Belgium), which has shown good results for far, intermediate, and near vision in terms of visual acuity, contrast sensitivity, and a photic phenomenon. ${ }^{24}$ However, to our knowledge, the LCA of eyes implanted with diffractive IOLs has not been measured in vivo.

In this study, we present in vivo LCA in the visible range (480 to $700 \mathrm{~nm}$ ) measured in pseudophakic patients bilaterally implanted with a hydrophilic trifocal diffractive IOL.

\section{PATIENTS AND METHODS}

LCA was obtained from psychophysical measurements of best focus and double-pass retinal image series at different wavelengths and viewing distances in 20 eyes from 10 patients bilaterally implanted with hydrophilic trifocal diffractive IOLs in each eye. Measurements were performed using a custom-developed polychromatic adaptive optics system.

\section{IOLS}

The implanted lenses were hydrophilic (26\% hudrophilic acrylic) multifocal diffractive IOLs. The Fine ion POD F IOL ${ }^{5,25}$ is an aspheric trifocal diffractive IOL, based on $100 \%$ diffractive technology, built with a combination of two bifocal diffractive patterns, of which one is for far and near vision and the other for far and intermediate vision. The IOL has a diffractive anterior surface entirely convoluted. By varying the step height of the diffractive structure of the IOL across the pupil, the energy distribution for different distances can be controlled. Indeed, the amount of energy directed to far vision focus is superior to that directed to intermediate and near vision focus with increasing apertures, by means of a gradual decrease in the height of diffractive steps from the center to the periphery, which is also the case for the refractive multifocal IOL. ${ }^{5}$ The combination of the two diffractive structures provides three useful focal distances: $0.00 \mathrm{D}$ for far vision, $+1.75 \mathrm{D}$ addition for intermediate vision, and +3.50 D addition for near vision. ${ }^{25}$

\section{Patients}

The study was conducted in both eyes of 10 patients (mean age: $66.70 \pm 3.25$ years; range: 51 to 75 years) (Table A, available in the online version of this article). Patients received a complete ophthalmic evaluation prior to enrollment in the study and surgery at IOA Madrid Innova Ocular (Madrid, Spain). The inclusion criteria for the study were good general health, no ocular pathology, no previous ocular surgery, IOL power between 16.00 and $26.00 \mathrm{D}$, corneal astigmatism of less than $1.25 \mathrm{D}$, bilateral implantation, and postoperative corrected distance visual acuity (CDVA) of worse than 0.7 decimal (20/30 Snellen or 0.16 logMAR). Surgeries in each eye were conducted with a time difference of less than 7 days.

All surgical procedures were performed by the same surgeon (FP) on an outpatient basis under topical anesthesia. A 2.2-mm corneal incision and a paracentesis were performed with a surgical knife. The anterior cap- 
sulotomy and nuclear fragmentation were created with the femtosecond laser under optical coherence tomography image control (CATALYS Precision System; Abbott Medical Optics, Inc., Santa Ana, CA). Phacoemulsification of the lens was performed with a commercial microsurgical system (Centurion Vision System; Alcon Laboratories, Inc., Fort Worth, TX). Two ophthalmic viscosurgical devices were used during the surgery: the cohesive Healon (Abbott Laboratories, Inc.) and the dispersive Amvisc (Bausch \& Lomb, Inc., Rochester, NY). The chosen IOL was then implanted in the capsular bag with a single-use injection system (Microset; PhysIOL). Following IOL implantation, all traces of ophthalmic viscosurgical devices were removed. In all cases, a capsular tension ring was inserted and all surgical procedures were performed using the computer-assisted cataract surgery system (CALLISTO Eye from Zeiss' Cataract Suite Markerless; Carl Zeiss, Jena, Germany). Postoperatively, patients were treated with a combination of antibiotic, corticosteroid, and anti-inflammatory drops (moxifloxacin, dexamethasone, and bromfenac) following our protocol.

All participants were acquainted with the nature and possible consequences of the study and provided written informed consent. All protocols met the tenets of the Declaration of Helsinki and had been previously approved by the Spanish National Research Council (CSIC) Bioethical Committee.

\section{Polychromatic AdaPtive Optics System}

Measurements were conducted in a custom-developed polychromatic adaptive optics system at the Visual Optics and Biophotonics Lab (Instituto de Óptica, CSIC), described in detail in previous publications, ${ }^{26-28}$ which allowed control of the aberrations of the system while performing both psychophysical settings of best focus and double-pass retinal imaging at different wavelengths.

The components of the system specifically used in the measurements were:

1. Illumination channel, with light coming from a supercontinuum laser source, allowed two independently filtered light fiber outputs (Visible-channel: 480, 532, 550, 650, and $700 \mathrm{~nm}$ and NIR channel: 780, 827, and $950 \mathrm{~nm}$ ). The laser power measured at the corneal plane ranged between 0.5 and $50 \mu \mathrm{W}$, within the American National Standards Institute safety limits at all wavelengths. ${ }^{29}$

2. Adaptive optics channel, consisting of the HartmannShack wavefront sensor (microlens array $40 \times 32$, 3.6-mm effective diameter) and the electromagnetic deformable mirror (52 actuators, 15-mm effective diameter, 50- $\mu \mathrm{m}$ stroke), which for the purposes of this study was used only to correct the aberrations of the optical system.

3. Double-pass retinal imaging channel (charge coupled device digital camera, 12-bit, monochrome, 6.7 $\times 6.7 \mu^{2}$ pixel size, $1,024 \times 1,280$ pixels), which allowed capture of aerial retinal images.

4. Pupil monitoring channel, consisting of a CMOS camera acquiring images of the pupil. The effective pupil diameter was limited to $4 \mathrm{~mm}$ with an artificial pupil. The patient's eye was aligned to the system (using an $\mathrm{x}-\mathrm{y}-\mathrm{z}$ stage) while visualizing the natural pupil in a monitor, using the line of sight as a reference.

5. Psychophysical channel, based on a digital micromirror device, placed in a conjugate retinal plane, and used to display visual stimuli $\left(1.62^{\circ}\right.$ angular subtend). The digital micromirror device was monochromatically illuminated with light coming from the supercontinuum laser source at different wavelengths. A holographic diffuser placed in the beam path breaks the coherence of the laser, providing a uniform illumination of the stimulus. The luminance of the stimulus was 20 to $25 \mathrm{~cd} / \mathrm{m}^{2}$ in the spectral range tested psychophysically (480 to 700 $\mathrm{nm}$ ), which was in the photopic region at all wavelengths $\left(>10 \mathrm{~cd} / \mathrm{m}^{2}\right)$.

In addition, a Badal system corrects for defocus and allows the psychophysical experiments. Two automatized shutters allow simultaneous illumination of the eye and the stimulus. All of the optoelectronic elements of the system were automatically controlled using custom-built software. The chromatic LCA of the system was measured and measurements were corrected by the calibrated LCA of the optical system, as described in detail in a previous publication. ${ }^{27}$

\section{EXPERIMENTS}

LCA was obtained from psychophysical and doublepass retinal image series for each of the tested wavelengths and distances. The best subjective focus for far vision was initially searched with the stimulus illuminated at a reference wavelength of $550 \mathrm{~nm}$ and set as zero. The following experiments were performed in the following order.

Experiment 1: Psychophysical Best Focus at Different Wavelengths and Distances. Patients adjusted their best subjective focus using the Badal system while viewing the stimulus illuminated with five different wavelengths in visible light $(480,532,555,650$, and $700 \mathrm{~nm})$. 
Patients were instructed to use the keyboards to move the Badal system toward the position where the stimulus (initially blurred), appeared sharp for the first time. Patients performed a trial before the experiment to become familiar with the test. The best focus settings were repeated three times for each wavelength. The different wavelengths were presented randomly. Measurements were performed at three different viewing distances simulated with the Badal system: 0.00 D for far vision, +1.75 D for intermediate vision, and +3.50 D for near vision.

Experiment 2: Double-Pass Retinal Image Series at Different Wavelengths and Distances. Throughfocus double-pass retinal aerial image series of a point source were collected at different wavelengths and different distances, whereas the Badal system corrected the subjective defocus of the patient at 555 $\mathrm{nm}$. Retinal aerial images were obtained while the Badal system was moved from -1.50 to $+1.50 \mathrm{D}$ in 0.25 -D steps (around the best subjective focus at 555 $\mathrm{nm}$ ). These measurements were obtained at three different wavelengths $(480,555$, and $700 \mathrm{~nm})$ and three different distances simulated with the Badal system (0.00 D for far vision, +1.75 D for intermediate vision, and $+3.50 \mathrm{D}$ for near vision).

All experiments were conducted under mydriasis (tropicamide 1\%, two drops 30 minutes prior to the beginning of the study and one drop every 1 hour). Each measurement session lasted approximately 4 hours.

\section{Data Analysis}

The best subjective foci at each wavelength in Experiment 1 were directly obtained from the automatic readings of the Badal optometer. The best foci at each wavelength in Experiment 2 were obtained by analysis of the through-focus retinal aerial image series for each wavelength. ${ }^{30}$ The best focus image from the series was obtained from the focus position corresponding to the image with minimum spread, from estimates of the width at half-height using custom routines written in Matlab (Mathworks, Natick, MA). Chromatic difference of focus curves were obtained from the data of best focus (either subjective from Experiment 1 or double-pass from Experiment 2). The LCA was obtained from the linear fittings to those curves.

Statistical analysis was performed with SPSS software (version 24.0; IBM Corporation, Armonk, NY) to test differences in the estimated LCA across experiments and conditions. A paired samples $t$ test was performed to analyze specific differences between conditions $(\mathrm{n}=10$, alpha $=0.05$, power $=0.80)$. A Wilcoxon signed-rank test was performed when the sampling conditions were not met.

\section{RESULTS}

One-month postoperative data for all patients are listed in Table A.

\section{Chromatic Difference of Focus from PSYCHOPHYSICAL AND DOUBLE-PASS TECHNIQUES}

Figure A (available in the online version of this article) shows the measured chromatic difference of focus for all measured wavelengths $(480,532,555,650$, and $700 \mathrm{~nm}$ ) and all distances (0.00 D for far vision = blue; $+1.75 \mathrm{D}$ for intermediate vision $=$ orange; and +3.50 $\mathrm{D}$ for near vision $=$ purple) from psychophysical measurements (Experiment 1). Lines represent linear fitting curves to the data. Except for patient 8, all patients consistently found their best focus at each wavelength for each viewing distance. All data are relative to best focus at far for $555 \mathrm{~nm}$.

Figure B (available in the online version of this article) shows the chromatic difference of focus for all measured wavelengths $(480,555$, and $700 \mathrm{~nm})$ and all distances $(0.00 \mathrm{D}$ for far vision = blue; $+1.75 \mathrm{D}$ for intermediate vision $=$ orange; and $+3.50 \mathrm{D}$ for near vision = purple) from through-focus double-pass retinal images series (Experiment 2). Lines represent linear fitting curves to the data. All data are relative to far best focus at $555 \mathrm{~nm}$.

Results show similar trends as Experiment 1, with all patients consistently finding their best focus at each wavelength for each viewing distance. At 555 $\mathrm{nm}$, the average focus shift is $+1.24 \mathrm{D}$ for the psychophysical measurements and $+0.85 \mathrm{D}$ for the doublepass measurements for the intermediate focus and $+2.97 \mathrm{D}$ for the psychophysical measurements and $+2.45 \mathrm{D}$ for the double-pass measurements for the near focus.

\section{LCA: DIFFERENCES ACROSS TECHNIQUES}

Figure C (available in the online version of this article) shows the LCA from psychophysical measurements (columns 1 and 2) and double-pass retinal series images (columns 3 and 4) in all patients and all eyes for all distances (far vision = blue; intermediate vision $=$ orange , near vision $=$ purple) in the visible range (480 to $700 \mathrm{~nm}$ ). Results show similar trends for all patients, except for patient 8 with higher LCA for far vision than for intermediate and near vision. The shift of best focus $(555 \mathrm{~nm})$ in this patient was 0.22 and $-0.38 \mathrm{D}$ for intermediate (right and left eye, respectively) and 1.71 and $0.56 \mathrm{D}$ for near (right and left eye, respectively), indicating that the patient may have misidentified the positions of best focus at different distances in the psychophysical measurements, leading to an overestimated LCA from psychophysical esti- 
mates at near in both eyes. Through-focus double-pass retinal images series are centered at the subjective best focus of each patient at each distance (far, intermediate, and near) for $555 \mathrm{~nm}$. For this specific patient, the misidentification of the subjective best focus led to an overestimation of LCA from double-pass retinal image series because the real best focus was not captured in those series. For these reasons, patient 8 was removed from the average computations.

Figure 1 shows the average LCA from psychophysical measurements and double-pass image series for far (blue), intermediate (orange), and near (purple) vision in the measured visible range (480 to $700 \mathrm{~nm}$ ), across patients (excluding patient 8). The LCA from psychophysical measurements was $0.82 \pm 0.05 \mathrm{D}$ for far vision, $0.27 \pm 0.15 \mathrm{D}$ for intermediate vision, and 0.15 $\pm 0.15 \mathrm{D}$ for near vision. The LCA from double-pass retinal image series was $0.72 \pm 0.10,0.19 \pm 0.15$, and 0.07 $\pm 0.17 \mathrm{D}$, respectively. LCA was significantly higher with both techniques $(P<.005)$ for far vision than for intermediate and near vision.

\section{DISCUSSION}

The dependence of the magnitude of LCA on distance is in agreement with previous studies that accounted for LCA in diffractive multifocal IOLs by optical simulations or on-bench measurements. Vega et al. ${ }^{22}$ compared the optical performance on-bench of diffractive multifocal IOLs with visible and near-infrared light and studied the variation in the energy balance between the different foci of the IOL, which is related to the dependence of the diffraction efficiency with wavelength. Multifocal IOLs often exhibit an asymmetrical energy distribution, normally biased to benefit the far focus against near/intermediate focus for larger punils, particularly in apodized designs (eg, the FineVisid_OD F IOL) and with large pupils. The previous on-bench optical study reported that under near-infrared illumination $(780 \mathrm{~nm}$ in their study, close to the $700 \mathrm{~nm}$ used in this study), optical performance of the IOLs was clearly biased in favor of far focus. In the current study, LCA of eyes implanted with the FineVision hydrophilic IOL obtained from experimental measurements in vivo (psychophysical measurements and double-pass retinal image series) was compared with LCA from on-bench measurements (personal communication, PhysIOL IOL + cornea and intraocular media, January 2017) and from optical simulations (performed for the current study using a Liou-Brennan eye model) in the same spectral range. We found on-bench and simulated LCA data showed similar distance dependence, with the LCA showing largest values at far and lower for near (on-bench: 1.54, 1.12 , and $0.23 \mathrm{D}$ for far, intermediate, and near vision,

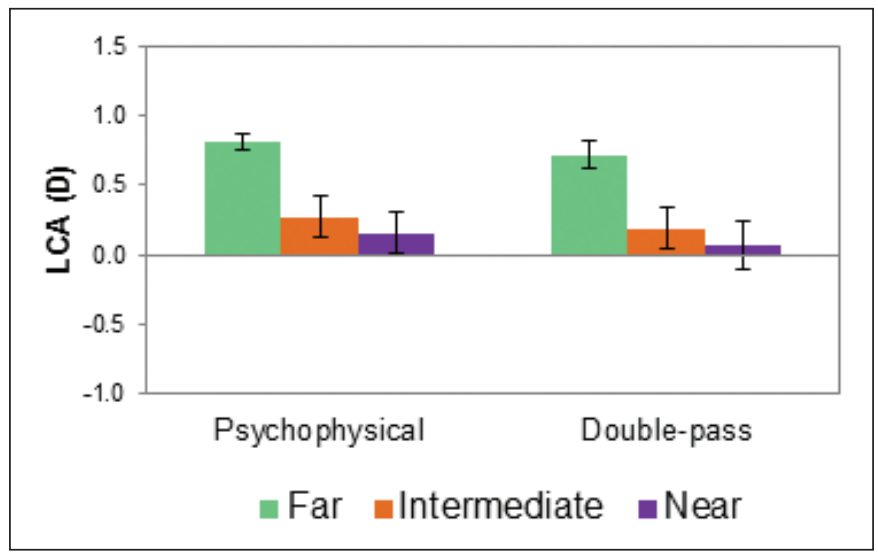

Figure 1. Average longitudinal chromatic aberration (LCA) from psychophysical measurements (left) and double-pass retinal image series (right) for all measured distances (far vision = blue; intermediate vision = orange; near vision $=$ purple). Error bars indicate inter-subject variability. $\mathrm{D}=$ diopters

respectively; simulated: $1.53,0.85$, and $0.23 \mathrm{D}$ for far, intermediate, and near vision, respectively). However, LCA values were higher than in vivo measurements, particularly for far vision.

It should be noted that the LCA values obtained from psychophysical methods and double-pass retinal image series for far vision were also lower than LCA values obtained in previous studies in young phakic eyes ${ }^{27}$ (1.52 and $0.95 \mathrm{D}$ from psychophysical and double-pass, respectively) and in eyes implanted with monofocal IOLs of the same material ${ }^{20}(1.21$ and $0.88 \mathrm{D}$ from psychophysical and double-pass, respectively) in the same spectral range (480 to $700 \mathrm{~nm}$ ). The decrease in LCA for the multifocal IOL at far with respect to the LCA for the monofocal IOL ${ }^{20}$ was found for both psychophysical data (0.39 D difference) and doublepass data (0.16 D difference). The presence of natural aberrations of the patients may have shifted LCA toward lower values. To understand the potential causes for LCA (far) reduction, we simulated the effect of different amounts of aberrations on the through-focus curves at various wavelengths and computed the LCA (far) in aberrated computer model eyes. We found that, in the presence of spherical aberration, the throughfocus curves were shifted and the peaks for far at two wavelengths (480 and $700 \mathrm{~nm}$ ) were closer, therefore reducing the effective LCA for far. For example, for 0.2 $\mu \mathrm{m}$ of spherical aberration, the LCA for far was $1.00 \mathrm{D}$, closer to the value measured psychophysically in this study (0.88 D) and significantly smaller than on-bench or the mentioned simulated data.

As expected from previous studies, double-pass LCA was systematically lower than psychophysical LCA. ${ }^{18,27}$ We attributed this shift to the spectral dependence on the retinal layer, where light is reflected 
that may compensate LCA partially by the lower wavelengths reflecting at more superficial layers (ie, near nerve fiber layer) and higher wavelengths in deeper retinal layers (ie, closer to the choroid). ${ }^{27}$ This hypothesis is also supported in the current study because the difference between the psychophysical and doublepass measurements remains fairly constant with distance ( 0.10 for far, 0.08 for intermediate, and $0.09 \mathrm{D}$ for near), although the differences were lower than those found for the monofocal IOL (0.33 D on average). A shift in the psychophysical LCA toward lower values may explain the relatively lower difference between psychophysical and double-pass LCA found in this study. A bias in the psychophysical best focus setting may be further imposed by the presence of multiple foci while performing the task. For example, a typical bias in best focus setting caused by the presence of other foci can be observed for patient 8 (right eye) in Figure A.

In general, patients provide systematic settings, as reflected by the relatively low inter-subject variability in the LCA $(0.11 \mathrm{D}$ in the psychophysical measurements and $0.14 \mathrm{D}$ in the double-pass measurements). LCA is mostly dependent on the dispersion properties of the intraocular media and the IOL material, and it is not expected to vary significantly in the population, as shown in reports of phakic ${ }^{31}$ or pseudophakic ${ }^{20}$ eyes. We did not find a significant correlation between LCA (for far) and IOL power $(P=.06$ for psychophysical LCA; $P=$ .11 for objective LCA). However, this inter-subject variability in LCA was larger than that found in patients implanted with monofocal IOLs of the same material ${ }^{20}$ $(0.08 \mathrm{D})$, which may be attributed to the observed impact of monochromatic aberrations on LCA with the multifocal IOLs. On the other hand, inter-eye differences in LCA in the same patients $(0.04$ for far and 0.05 for near psychophysical and 0.08 for far and -0.09 for near double-pass) were lower than inter-subject differences ( 0.05 for far, 0.15 for near psychophysical, and 0.10 for far and 0.15 for near double-pass), supporting the reproducibility of the data and suggesting some (small) contribution of the individual eye biometry, lens geometry, and individual intraocular media dispersion properties to LCA and, in particular, the role of aberrations (more likely to show higher inter-subject than inter-eye variability) on the LCA, as shown.

The ability to measure LCA in vivo allows quantifying retinal image quality in polychromatic eyes. Previous studies have reported the importance of the monochromatic and polychromatic aberration interactions in image quality in white light. Diffractive IOLs in which the diffractive component can modulate chromatic aberration introduce another variable that can be controlled to fine tune chromatic aberration. In this study, we found that LCA was reduced for intermediate or far, but a specific design can also minimize chromatic aberration for near. Correction of chromatic aberration opens a potential new avenue for improvement of retinal image quality, provided that balance with monochromatic aberration or the simultaneous vision in multifocal lens designs is not compromised.

\section{AUTHOR CONTRIBUTIONS}

Study concept and design (MV, SM); data collection ( $M V, A G-R$, $V A, N G, F P)$; analysis and interpretation of data (MV, CD, SM); writing the manuscript ( $M V, S M$ ); critical revision of the manuscript ( $M V$, $A G-R, C D, V A, N G, F P, S M$ ); statistical analysis (MV); administrative, technical or material support ( $M V, S M)$; supervision (MV, $S M$ )

\section{REFERENCES}

1. Charman WN. Developments in the correction of presbyopia II: surgical approaches. Ophthalmic Physiol Optics. 2014;34:397426.

2. Maxwell WA, Lane SS, Zhou F. Performance of presbyopiacorrecting intraocular lenses in distance optical bench tests. J Cataract Refract Surg. 2009;35:166-171.

3. Kim MJ, Zheleznyak L, Macrae S, Tchah H, Yoon G. Objective evaluation of through-focus optical performance of presbyopiacorrecting intraocular lenses using an optical bench system. $J$ Cataract Refract Surg. 2011;37:1305-1312.

4. Pepose JS, Wang D, Altmann GE. Comparison of through-focus image sharpness across five presbyopia-correcting intraocular lenses. Am J Ophthalmol. 2012;154:20-28 e21.

5. Gatinel D, Houbrechts Y. Comparison of bifocal and trifocal diffractive and refractive intraocular lenses using an optical bench. J Cataract Refract Surg. 2013;39:1093-1099.

6. Vega F, Alba-Bueno F, Millán MS. Energy distribution between distance and near images in apodized diffractive multifocal intraocular lenses. Invest Ophthalmol Vis Sci. 2011;52:56955701.

7. Vega F, Alba-Bueno F, Millán MS, Váron C, Gil MA, Buil JA. Halo and through-focus performance of four diffractive multifocal intraocular lenses. Invest Ophthalmol Vis Sci. 2015;56:39673975.

8. Akondi V, Pérez-Merino P, Martinez-Enriquez E, et al. Evaluation of the true wavefront aberrations in eyes implanted with a rotationally asymmetric multifocal intraocular lens. J Refract Surg. 2017;33:257-265.

9. Gatinel D. Limited accuracy of Hartmann-Shack wavefront sensing in eyes with diffractive multifocal IOLs. J Cataract Refract Surg. 2008;34:528.

10. Negishi K, Ohnuma K, Hirayama N, et al. Effect of chromatic aberration on contrast sensitivity in pseudophakic eyes. Arch Ophthalmol. 2001;119:1154-1158.

11. Vinas M, Dorronsoro C, Garzón N, Poyales F, Marcos S. In vivo subjective and objective longitudinal chromatic aberration after bilateral implantation of the same design of hydrophobic and hydrophilic intraocular lenses. J Cataract Refract Surg. 2015;41:2115-2124.

12. Artal P, Manzanera S, Piers P, Weeber H. Visual effect of the combined correction of spherical and longitudinal chromatic aberrations. Opt Express. 2010;18:1637-1648. 
13. Siedlecki D, Józwik A, Zajac M, Hill-Bator A, Turno-Krecicka A. In vivo longitudinal chromatic aberration of pseudophakic eyes. Optom Vis Sci. 2014;91:240-246.

14. Weeber HA, Piers PA. Theoretical performance of intraocular lenses correcting both spherical and chromatic aberration. JRefract Surg. 2012;28:48-52.

15. Castignoles F, Flury M, Lepine T. Comparison of the efficiency, MTF and chromatic properties of four diffractive bifocal intraocular lens designs. Opt Express. 2010;18:5245-5256.

16. Ravikumar S, Bradley A, Thibos LN. Chromatic aberration and polychromatic image quality with diffractive multifocal intraocular lenses. J Cataract Refract Surg. 2014;40:1192-1204.

17. Zhao H, Mainster MA. The effect of chromatic dispersion on pseudophakic optical performance. Br J Ophthalmol. 2007;91:12251229.

18. Nagata T, Kubota S, Watanabe I, Aoshima S. Chromatic aberration in pseudophakic eyes [article in Japanese]. Nippon Ganka Gakkai Zasshi. 1999;103:237-242.

19. Bobier CW, SivakJG. Chromoretinoscopy. Vision Res. 1978;18:247250.

20. Pérez-Merino P, Dorronsoro C, Llorente L, Durán S, JiménezAlfaro I, Marcos S. In vivo chromatic aberration in eyes implanted with intraocular lenses. Invest Ophthalmol Vis Sci. 2013;54:2654-2661.

21. Nakajima M, Hiraoka T, Yamamoto $T$, et al. Differences of longitudinal chromatic aberration (LCA) between eyes with intraocular lenses from different manufacturers. PLoS One. 2016;11:e0156227.

22. Vega F, Millan MS, Vila-Terricabras N, Alba-Bueno F. Visible versus near-infrared optical performance of diffractive multifocal intraocular lenses. Invest Ophthalmol Vis Sci. 2015;56:7345-7351.
23. Millán MS, Vega F, Ríos-López I. Polychromatic Image performance of diffractive bifocal intraocular lenses: longitudinal chromatic aberration and energy efficiency. Invest Ophthalmol Vis Sci. 2016;57:2021-2028.

24. Carballo-Alvarez J, Vazquez-Molini JM, Sanz-Fernandez JC, et al. Visual outcomes after bilateral trifocal diffractive intraocular lens implantation. BMC Ophthalmol. 2015;15:26.

25. Gatinel D, Pagnoulle C, Houbrechts Y, Gobin L. Design and qualification of a diffractive trifocal optical profile for intraocular lenses. J Cataract Refract Surg. 2011;37:2060-2067.

26. Vinas M, Dorronsoro C, Gonzalez V, Cortes D, Radhakrishnan A, Marcos S. Testing vision with angular and radial multifocal designs using Adaptive Optics. Vision Res. 2016:132:8596.

27. Vinas M, Dorronsoro C, Cortes D, Pascual D, Marcos S. Longitudinal chromatic aberration of the human eye in the visible and near infrared from wavefront sensing, double-pass and psychophysics. Biomed Opt Exp. 2015;6:948-962.

28. Vinas M, Dorronsoro C, Radhakrishnan A, et al. Comparison of vision through surface modulated and spatial light modulated multifocal optics. Biomed Opt Exp. 2017;8:2055-2068.

29. Delori FC, Webb RH, Sliney DH. Maximum permissible exposures for ocular safety (ANSI 2000), with emphasis on ophthalmic devices. J Opt Soc Am A Opt Image Sci Vis. 2007;24:12501265.

30. Marcos S, Moreno E, Navarro R. The depth-of-field of the human eye from objective and subjective measurements. Vision Res. 1999;39:2039-2049.

31. Charman WN, Jennings JA. Objective measurements of the longitudinal chromatic aberration of the human eye. Vision Res. 1976;16:999-1005 


\begin{tabular}{|c|c|c|c|c|c|c|c|c|c|}
\hline \multicolumn{10}{|c|}{$\begin{array}{c}\text { TABLE } A \\
\text { 1-Month Postoperative Clinical Data }\end{array}$} \\
\hline Patient & Sex & Age $(y)$ & Eye & IOL Power (D) & Sphere (D) & Cylinder (D) & Axis $\left({ }^{\circ}\right)$ & UDVA & CDVA \\
\hline \multirow[t]{2}{*}{1} & $M$ & 69 & $\mathrm{R}$ & 19.50 & -0.50 & -0.50 & 110 & $20 / 30$ & $20 / 20$ \\
\hline & & & L & 19.50 & 0.50 & -0.50 & 70 & $20 / 25$ & $20 / 20$ \\
\hline \multirow[t]{2}{*}{2} & $\mathrm{~F}$ & 69 & $\mathrm{R}$ & 24.00 & 0.00 & 0.00 & - & $20 / 20$ & $20 / 20$ \\
\hline & & & L & 24.00 & 0.00 & 0.00 & - & $20 / 19$ & $20 / 20$ \\
\hline \multirow[t]{2}{*}{3} & $\mathrm{~F}$ & 71 & $R$ & 23.50 & 0.50 & 0.00 & - & $20 / 25$ & $20 / 25$ \\
\hline & & & L & 23.50 & 0.00 & 0.00 & - & $20 / 20$ & $20 / 20$ \\
\hline \multirow[t]{2}{*}{4} & M & 75 & $\mathrm{R}$ & 20.00 & 0.00 & -1.25 & 70 & $20 / 50$ & $20 / 20$ \\
\hline & & & L & 20.00 & 0.00 & -1.00 & 105 & $20 / 30$ & $20 / 20$ \\
\hline \multirow[t]{2}{*}{5} & M & 69 & $\mathrm{R}$ & 22.00 & 0.00 & 0.00 & - & $20 / 20$ & $20 / 20$ \\
\hline & & & L & 22.50 & 0.00 & 0.00 & - & $20 / 20$ & $20 / 20$ \\
\hline \multirow[t]{2}{*}{6} & $\mathrm{~F}$ & 51 & $\mathrm{R}$ & 16.50 & 0.00 & 0.00 & - & $20 / 20$ & $20 / 20$ \\
\hline & & & L & 17.00 & 0.00 & 0.00 & - & $20 / 20$ & $20 / 20$ \\
\hline \multirow[t]{2}{*}{7} & $\mathrm{~F}$ & 65 & $\mathrm{R}$ & 26.00 & -0.75 & -0.50 & 130 & $20 / 30$ & $20 / 20$ \\
\hline & & & L & 26.00 & 0.25 & -0.50 & 70 & $20 / 30$ & $20 / 20$ \\
\hline \multirow[t]{2}{*}{8} & $\mathrm{~F}$ & 69 & $\mathrm{R}$ & 23.50 & 0.00 & 0.00 & - & $20 / 20$ & $20 / 20$ \\
\hline & & & L & 23.50 & 0.00 & 0.00 & - & $20 / 20$ & $20 / 20$ \\
\hline \multirow[t]{2}{*}{9} & $\mathrm{~F}$ & 62 & $\mathrm{R}$ & 22.50 & 0.00 & 0.00 & - & $20 / 20$ & $20 / 20$ \\
\hline & & & L & 22.00 & 0.50 & -0.50 & 170 & $20 / 25$ & $20 / 20$ \\
\hline \multirow[t]{2}{*}{10} & $\mathrm{~F}$ & 67 & $R$ & 26.00 & 0.00 & 0.00 & - & $20 / 20$ & $20 / 20$ \\
\hline & & & L & 28.00 & 0.75 & -0.75 & 70 & $20 / 30$ & $20 / 20$ \\
\hline
\end{tabular}




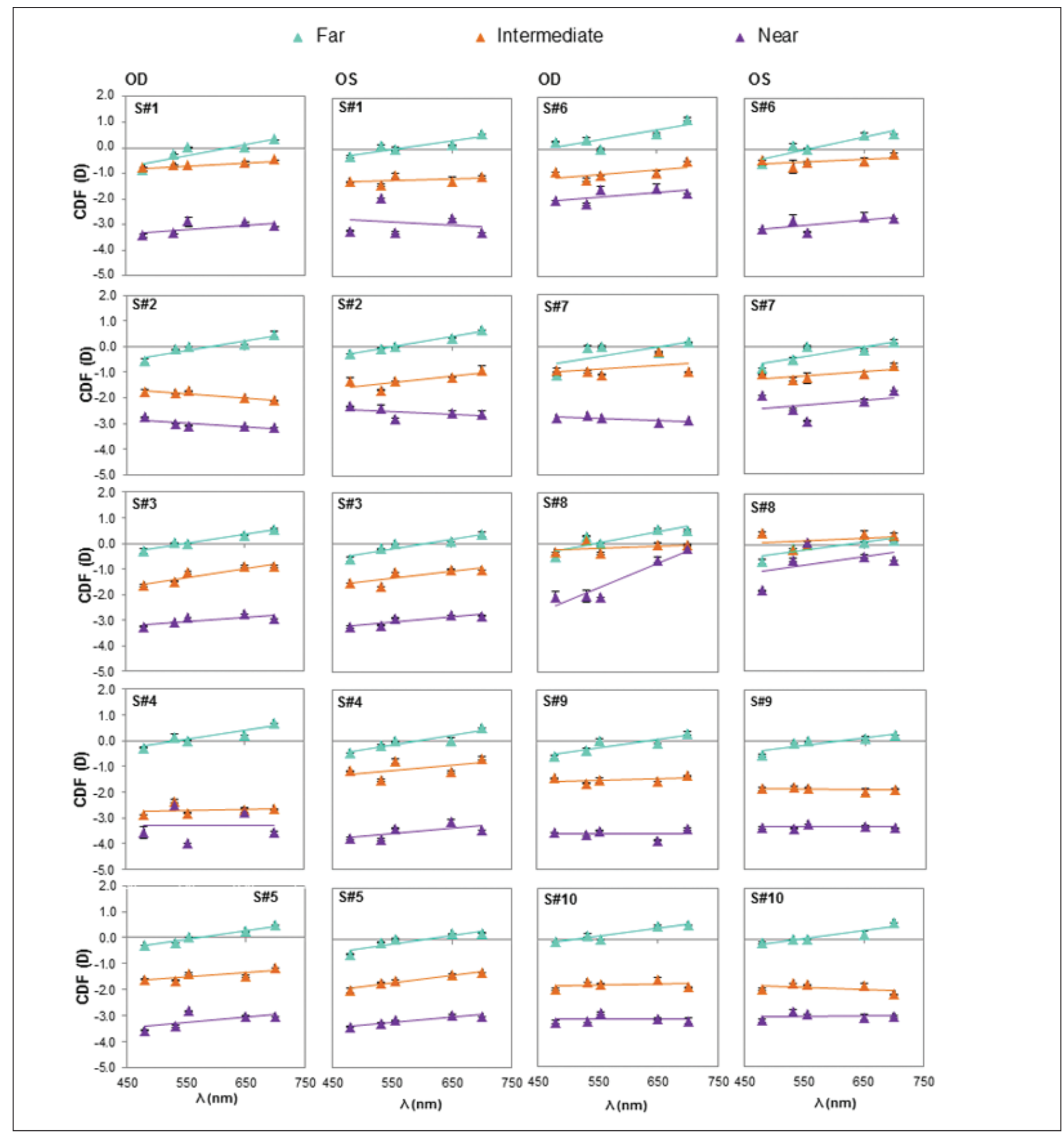

Figure A. Chromatic difference of focus (CDF) from psychophysical best focus of monochromatic stimuli, for all patients, all measured wavelengths (480, 532, 555, 650 \& $700 \mathrm{~nm}$ ), and all distances (far vision, blue; intermediate vision, orange; near vision, purple). Error bars stand for standard deviation across measurements. $O D=$ right eye, $O S=$ left eye; $D=$ diopters 


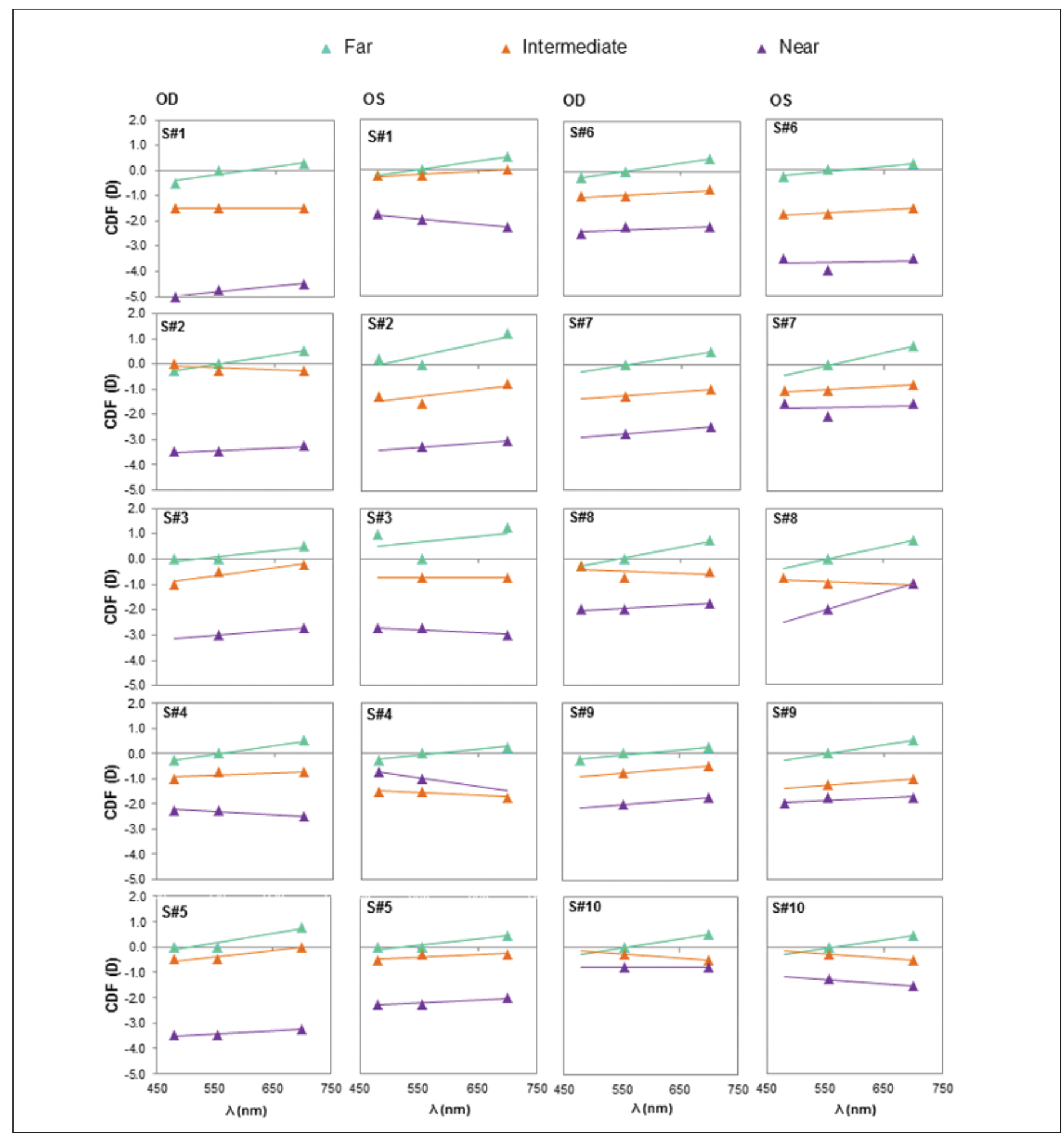

Figure B. Chromatic difference of focus (CDF) from through-focus double-pass retinal images series, for all patients, all measured wavelengths (480, 555 , and $700 \mathrm{~nm}$ ), and all distances (far vision = blue; intermediate vision = orange; near vision $=$ purple). OD = right eye, OS $=$ left eye; $\mathrm{D}=$ diopters 

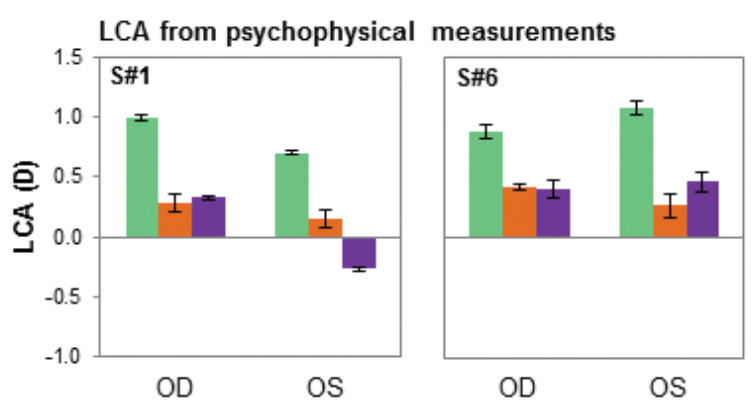

LCA from double-pass retinal image series
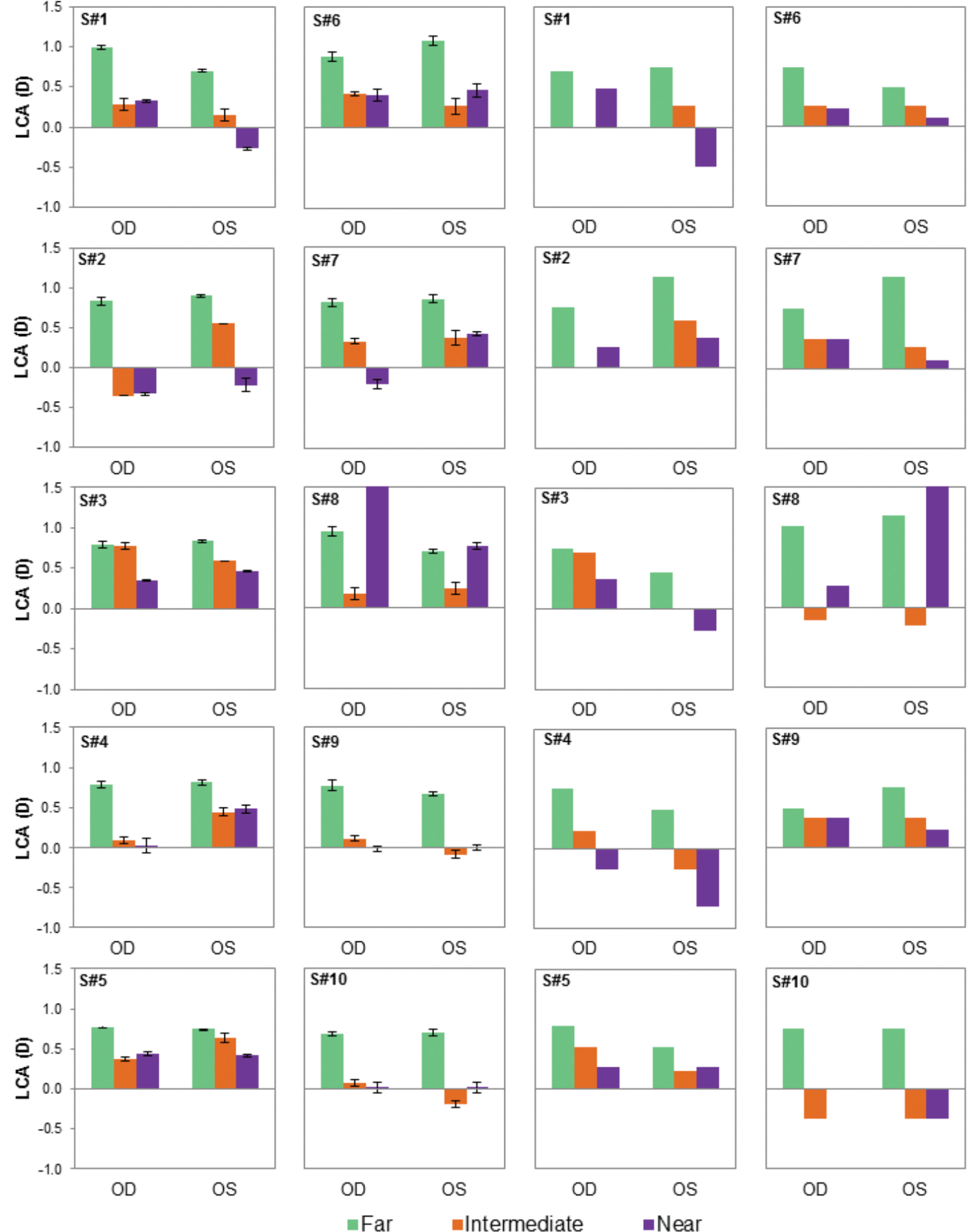

Figure C. Longitudinal chromatic aberration (LCA) from psychophysical measurements (columns 1 and 2) and from double-pass retinal image series (columns 3 and 4) for all patients, all measured wavelengths $(480,555$, and $700 \mathrm{~nm}$ ), and all distances (far vision $=$ blue; intermediate vision $=$ orange; near vision $=$ purple). Error bars indicate standard deviation across psychophysical measurements. $\mathrm{OD}=$ right eye, $\mathrm{OS}=$ left eye; $\mathrm{D}=$ diopters 